\title{
Biologia reprodutiva e guilda de visitantes florais de Pseudobombax marginatum (Malvaceae)
}

Reproductive biology and flower visitors guild of Pseudobombax marginatum (Malvaceae)

\author{
Izaac Damasceno Pequeno ${ }^{1}$, Natan Messias Almeida ${ }^{2,4}$ \& José Alves Siqueira Filho ${ }^{1,3}$
}

\begin{abstract}
Resumo
No Brasil, ocorrem 120 espécies do gênero Pseudobombax em diferentes formações vegetacionais. Espécies de Bombacoideae apresentam flores com antese noturna, visitadas por morcegos e esfingídeos. Objetivou-se investigar a biologia reprodutiva de $P$. marginatum, e conhecer relações entre planta e visitantes. O estudo foi desenvolvido em uma área de caatinga, no município de Afrânio, Pernambuco. Analisou-se a fenologia, biologia floral, comportamento dos visitantes e sistema reprodutivo. P. marginatum apresenta características de flores quiropterófilas, apesar de não receber visitas de morcegos, parecendo estar relacionado ao processo de fragmentação florestal ocorrente na área de estudo. A espécie floresceu entre maio e julho, recebendo visitas de esfingídeos, abelhas, vespas e aves. A produção de néctar foi elevada com baixa concentração de açúcares. Apenas na polinização cruzada obteve-se sucesso, devido à presença de mecanismos de incompatibilidade. P. marginatum apresenta-se em situação crítica quanto à sua regeneração, devido à carência de serviços de polinização eficientes, provavelmente ocasionados pela perturbação na área e ausência de quirópteros visitantes. Palavras-chave: fragmentação florestal, serviço de polinização, mecanismos de incompatibilidade.

Abstract

In Brazil, the genus Pseudobombax is represented by some 120 species occurring in different vegetation types. Bombacoideae species have nocturnal flowers and are bat and hawkmoth visited. The aim of the work was to investigate the reproductive biology of $P$. marginatum, and to describe the plant-floral visitors relationships. The study was carried out in a Caatinga area in Afrânio municipality, Pernambuco state. Phenology, floral biology, flower-visitor behavior and reproductive system were analyzed. P. marginatum has chiropterophilous flowers, although it is not visited by bats, a fact possibly related to the forest fragmentation process in the study area. The flowering period of the species was from May to July, - floral visitors were hawkmoths, bees, wasps and birds. Nectar production was high with low sugar concentration. Reproductive success was obtained only in cross pollination, due to the incompatibility mechanism. P. marginatum presents a critical situation for regeneration due to the lack of efficient pollination services, caused probably by forest fragmentation in the study area and the lack of chiropterans visitors.
\end{abstract}

Key words: forest fragmentation, pollination service, incompatibility mechanism.

\section{Introdução}

A subfamília Bombacoideae está amplamente distribuída nas regiões tropicais, com 30 gêneros agrupando cerca de 200 espécies (Robyns 1963; Baum et al. 2004). No Brasil está representada por 14 gêneros com cerca de 120 espécies, ocorrentes em diferentes formações vegetacionais (Barroso et al. 1978), com centros de diversidade nas regiões Norte e Nordeste (Robyns 1963). Atualmente, Bombacoideae está inserida na família Malvaceae s.l. (Alverson et al. 1999; CarvalhoSobrinho \& Queiroz 2011; Souza \& Lorenzi 2012) e morfologicamente caracteriza-se pelo porte predominantemente arbóreo, folhas palmadamente compostas e digitadas e pelos frutos do tipo cápsula (Carvalho-Sobrinho \& Queiroz 2011).

\footnotetext{
${ }^{1}$ Universidade Federal do Vale do São Francisco, Rod. BR 407, 12, Lote 543, Projeto de Irrigação Nilo Coelho, C1, Petrolina, PE, Brasil.

${ }^{2}$ Universidade Estadual de Alagoas, Rod. AL 115, Zona Rural, Palmeira dos Índios, AL, Brasil.

${ }^{3}$ Centro de Referência para a Recuperação de Áreas Degradadas da Bacia Hidrográfica do Rio São Francisco (CRAD), Universidade Federal do Vale do São Francisco (UNIVASF), Distrito de Irrigacão Senador Nilo Coelho, 56300-990 Petrolina, PE, Brasil.

${ }^{4}$ Autor para correspondência: natanmessias@yahoo.com.br
} 
Pseudobombax Dugand é um gênero Neotropical (Robyns 1963) representado por 27 espécies, principalmente em áreas sob clima estacional nos Biomas Cerrado e Caatinga. $\mathrm{Na}$ região do semiárido nordestino, o gênero Pseudobombax é o mais representativo da subfamília em número de espécies (CarvalhoSobrinho \& Queiroz 2011).

Estudos sobre sistema de polinização envolvendo espécies de Pseudobombax foram realizados em diversos ecossistemas com $P$. ellipticum (H.B.K.) Dugand na Floresta baixa caducifólia no México (Eguiarte et al. 1987), P. tomentosum (Mart. \& Zucc.) A. Robyns em área de Cerrado (Gribel 1988), P. grandiflorum (Cav.) A. Robyns em Mata atlântica (Fischer et al. 1992) e P. munguba (Mart. et Zucc.) Dugand na Amazônia (Gribel \& Gibbs 2002). No entanto, apesar da representatividade do grupo no semiárido não existem registros na literatura sobre o estudo da biologia floral e reprodutiva de representantes do gênero na Caatinga.

Características da biologia floral, polinização e sistema reprodutivo observadas para a espécie estudada, são comuns para espécies da subfamília Bombacoideae. Estudos relacionados a biologia floral das espécies do gênero Pseudobombax, mostram que as mesmas apresentam como atributos comuns flores grandes com antese noturna, variando de alvas a lilases, com hercogamia pronunciada e ausência de guias de néctar e até mesmo de néctar, como é o caso de $P$. munguba (Baker et al. 1998; Eguiarte et al. 1987; Faegri \& Pijl 1979; Fischer et al. 1992; Gribel 1988; Gribel \& Gibbs 2002; Silva \& Peracchi 1995; Yamamoto et al. 2007; Teixeira 2010; Zortéa 2003). Estas características são comuns a espécies esfingófilas e quiropterófilas, o que corrobora com o observado em estudos com espécies de Pseudobombax (Eguiarte et al. 1987; Gribel 1988; Fischer et al. 1992; Silva \& Peracchi 1995; Gribel \& Gibbs 2002; Zortéa 2003; Yamamoto et al. 2007; Teixeira 2010).

$\mathrm{O}$ estudo e consequente conhecimento da biologia reprodutiva de espécies vegetais são de fundamental importância em programas de conservação e manejo das mesmas, principalmente quando estas espécies estão expostas a pressões impostas pela fragmentação do hábitat como carência de polinizadores específicos e redução no número de indivíduos da população, que podem levar a limitação polínica (Harder \& Aizen 2010; Freitas et al. 2010). Estudos demonstram efeitos negativos destas pressões nas interações entre plantas e animais, e consequentemente no sucesso reprodutivo das espécies vegetais (Lennartsson 2000; Aizen \& Feinsinger 2003).

O presente estudo teve como objetivos, descrever a biologia floral e fenologia de floração, investigar aspectos da biologia reprodutiva de $P$. marginatum, e estudar a interação entre esta espécie vegetal e seus visitantes florais, em um ambiente de caatinga fragmentado.

\section{Material e Métodos}

Local de estudo

Os estudos de campo foram desenvolvidos durante os períodos de fevereiro 2006 a agosto de 2007 e maio de 2008 a novembro de 2008 no povoado de Caboclo (8²8'56,4' $\mathrm{S}, 40^{\circ} 56^{\prime} 6,9^{\prime}$ 'W, $588 \mathrm{~m}$ de altitude), município de Afrânio, Pernambuco. As observações foram feitas mensalmente durante todo o estudo, sendo os indivíduos acompanhados diariamente durante o período de floração.

A área de estudo apresenta vegetação de Caatinga com clima predominantemente quente e semiárido, precipitação anual variando entre $500 \mathrm{e}$ $800 \mathrm{~mm}$ (Velloso et al. 2002), solos do tipo Neossolo e Argissolo Vermelho (Embrapa 1999). A área está inserida na porção "Oeste de Pernambuco", que é considerada de alta importância biológica para conservação da Caatinga, em razão da ocorrência de espécies vegetais e animais endêmicas e ameaçadas de extinção (MMA 2002).

\section{Espécie estudada}

Pseudobombax marginatum apresenta porte arbóreo medindo entre 6 e $14 \mathrm{~m}$ e tronco com estrias longitudinais esverdeadas (Siqueira-Filho et al. 2009). Possui flores do tipo pincel, com antese noturna. Estudos realizados anteriormente na área mostram que a floração de $P$. marginatum ocorre entre os meses de maio e agosto e a frutificação entre agosto e novembro (SiqueiraFilho et al. 2009).

O material botânico coletado foi incorporado ao Herbário da Universidade Federal do Vale do São Francisco (HVASF-282).

\section{Fenologia e biologia floral}

Durante o período de estudo, os indivíduos de P. marginatum foram observados mensalmente $(\mathrm{n}=$ 10 indivíduos), acompanhados diariamente durante a floração $(\mathrm{n}=23$ indivíduos) e semanalmente durante a frutificação $(\mathrm{n}=23$ indivíduos). Em campo, foram registradas informações como cor 
e odor das flores, duração e período de antese, receptividade do estigma, volume e concentração de néctar e disponibilidade de pólen, através inspeções visuais no campo. As flores coletadas $(n=15)$ foram levadas ao laboratório, onde o número, disposição das peças florais e tamanho das estruturas, utilizando paquímetro digital $(0005 \% / 0,01 \mathrm{~mm})$, foram avaliados. Seguindo as classificações de Faegri \& Pijl (1979), foi determinada a síndrome floral da espécie e o padrão fenológico foi inferido conforme as classificações de Gentry (1974) e Newstrom et al. (1994).

Os botões em pré-antese foram isolados com sacos de "voile" por cerca de 12 horas (18 $\mathrm{h}-8 \mathrm{~h}$ ), para quantificar o volume acumulado e a concentração de açúcares no néctar. Para isso foram utilizados, respectivamente, microsseringas (Hamilton ${ }^{\circledR} 50 \mu \mathrm{l}$ ) e refratômetro de bolso (escala $0-32 \%)$.

A receptividade do estigma foi testada com $\mathrm{H}_{2} \mathrm{O}_{2}$ (Peróxido de Hidrogênio), ao longo da antese e observada em campo com o auxílio de uma lente de aumento (Galen \& Plowright 1987).

\section{Visitantes florais}

Foram registrados o horário, a duração, a freqüência, número de visitas e resultado das visitas (polinização, quando os visitantes contatavam anteras e estigmas no momento da visita; ou pilhagem, quando não havia contato com anteras e estigma). Estas observações foram realizadas em indivíduos focais, durante 20 dias não consecutivos, resultando em $60 \mathrm{~h}$ de esforço, além de observações ocasionais em períodos de realização dos tratamentos do sistema reprodutivo e análise fenológica. Os principais visitantes foram fotografados e/ou capturados para posterior identificação por especialistas.

Os insetos foram montados a seco e depositados na Coleção do Laboratório de Botânica e Conservação da Biodiversidade - UNIVASF.

\section{Sistema reprodutivo}

Foram realizados os tratamentos de 1) polinização cruzada: em flores emasculadas (n =16) nas quais foram realizadas as polinizações manuais com pólen originado de outro indivíduo, e subseqüente isolamento; 2) Agamospermia: botões em pré-antese $(\mathrm{n}=16)$ foram emasculados e em seguida isolados; 3) Autopolinização espontânea: botões isolados $(\mathrm{n}=25)$ durante a pré-antese; 4) Autopolinização manual: flores isoladas $(\mathrm{n}=15)$ durante a pré-antese e polinizadas manualmente, com pólen produzido pela própria flor, no momento da abertura (Goldenberg \& Shepherd 1998). As flores foram isoladas com sacos de "voile", amarrados nas extremidades com fio de náilon, de forma a permitir a abertura natural da flor. Para obtenção do número de frutos formados naturalmente (controle) as flores $(n=30)$ foram expostas a ação dos visitantes durante o período de antese.

Posteriormente, havendo formação de frutos, os mesmos foram contabilizados bem como as sementes.

\section{Resultados}

\section{Fenologia e biologia floral}

Os indivíduos de Pseudobombax marginatum estudados apresentam porte arbóreo, com cinco a sete metros de altura. Apresentam inflorescências com flores solitárias ou em cimeiras 3-flores, dispostas no ápice dos ramos. As flores são actinomorfas, monóclinas, polistêmones, do tipo pincel (Fig. 1a). O receptáculo floral é formado por glândulas nectaríferas dispostas em um anel contínuo de coloração rósea na sua face externa. A porção interna do cálice forma a câmara nectarífera, resultado da união das sépalas em tubo. As pétalas apresentam face interna alva, com tricomas simples e a face externa amarronzada, com tricomas tufosos. Os estames se dispõem de forma hemisférica, conados em um tubo estaminal e as anteras apresentam deiscência longitudinal. O gineceu possui ovário súpero, sincárpico, 5-carpelar, subereto, persistente. O estigma é 5-lobado e localiza-se acima do nível das anteras de modo a provocar uma separação espacial entre estas estruturas, caracterizando a hercogamia. Os frutos são do tipo cápsula lenhosa 5-valvar. As sementes são numerosas, medindo entre seis e sete $\mathrm{mm}$ (n $=30$ ) de comprimento, ovais, marrom-escuras, com estrias longitudinais brancas, e apresentam aderida a elas uma paina que varia de esbranquiçada a dourada. Dados acerca das dimensões das estruturas florais (androceu, gineceu, tubo estaminal e glândulas nectaríferas) de $P$. marginatum encontram-se na Tabela 1.

Pseudobombax marginatum apresentou o padrão de floração anual (Newstron et al. 1994) e estratégia fenológica do tipo disponibilidade regular (Gentry 1974), florescendo entre os meses de maio e julho, período de transição entre as estações chuvosa e seca, perdendo as folhas imediatamente antes da floração. As flores apresentaram antese noturna, em número de 14,9 $\pm 11,25$ flores/dia $(n=15)$, com as pétalas se retorcendo no início da antese. 
Tabela 1 - Número e dimensões das flores e estruturas florais de Pseudobombax marginatum (A. St.-Hil., Juss. \& Cambess.) A. Robyns (Malvaceae), em Caboclo, Afrânio, Pernambuco.

Table 1 - Number and size of flowers and floral structures of Pseudobombax marginatum (A. St.-Hil., Juss. \& Cambess.) A. Robyns (Malvaceae), in Caboclo, Afrânio, Pernambuco.

\begin{tabular}{lccc}
\hline \multirow{2}{*}{ Estruturas } & \multicolumn{3}{c}{ Número e dimensões } \\
\cline { 2 - 4 } & $\mathbf{N}^{\mathbf{0}}$ & Comprimento (cm) & Diâmetro (mm) \\
\hline Flor & - & $14,85 \pm 0,78$ & - \\
Receptáculo (glândulas nectaríferas) & $12 \pm 0,48$ & - & - \\
Cálice (tubo) & & $20,19 \pm 1,45$ & - \\
Androceu & $442 \pm 29,01$ & - & - \\
Estames & - & $9,36 \pm 1,98$ & $10,97 \pm 1,96$ \\
Tubo estaminal & - & $12,45 \pm 2,05$ & - \\
Gineceu & - & $14,97 \pm 1,08$ & \\
\hline
\end{tabular}

A antese das flores é explosiva de $P$. marginatum é explosiva e iniciou-se por volta das $18 \mathrm{~h}$, com o afastamento das pétalas de modo a torná-la convexa, propiciando a liberação inicial dos estames menores. A antese se prolongou até às $9 \mathrm{~h}$ do dia seguinte, horário em que todas as flores já se apresentavam murchas. O estigma permaneceu receptivo e as anteras funcionais, com pólen exposto durante todo o período da antese. $\mathrm{O}$ volume acumulado de néctar aferido foi de 293,22 $\mu \mathrm{L} \pm 149,36(\mathrm{n}=10)$ por flor e a concentração média de açúcares foi de 14,57\% $03,08(\mathrm{n}=10)$. As flores, ao receber qualquer agitação, liberaram uma "nuvem" de pólen que se depositava nas pétalas, cálice e filetes.

Ao final da antese, as flores apresentaram-se esmaecidas, com estigma e anteras apresentando coloração amarronzada e o androceu desprendendose do receptáculo. O odor das flores é forte e desagradável.
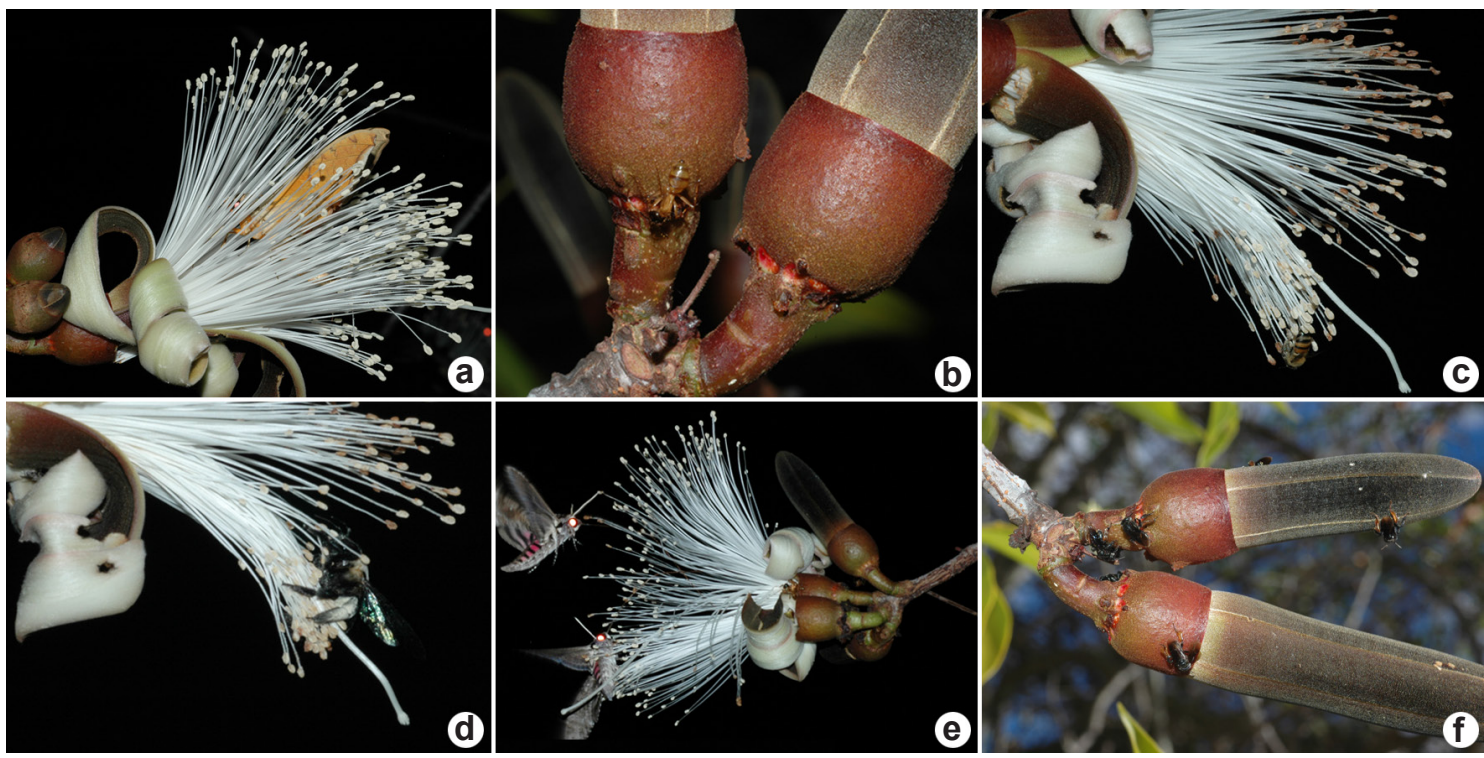

Figura 1 - Visitantes florais de Pseudobombax marginatum em uma área de Caatinga em Caboclo, Afrânio, Pernambuco - a. Protambulix strigilis (Sphingidae); b. Camponotus sp. (Formicidae), patrulhando botões florais; c. Apis mellifera (Apidae), pilhando pólen; d. Xylocopa (Neoxylocopa) grisescens (Apidae), coletando pólen; e. Agrius cingulata (Sphingidae); f. Trigona spinipes (Apidae). Fotos: a, c, d, e por J.A. Siqueira Filho; b, f por F.S.E. Santo.

Figure 1 - Floral visitors of Pseudobombax marginatum in an área of Caatinga in Caboclo, Afrânio, Pernambuco - a. Protambulix strigilis (Sphingidae); b. Camponotus sp. (Formicidae), patrolling flower buds; c. Apis mellifera (Apidae), pollen thieve; d. Xylocopa (Neoxylocopa) grisescens (Apidae), collecting pollen;e. Agrius cingulata (Sphingidae); f. Trigona spinipes (Apidae). Photos: a, c, d, e by J.A. Siqueira Filho; b, f by F.S.E. Santo. 
Tabela 2 - Visitantes florais de Pseudobombax marginatum (A. St.-Hil., Juss. \& Cambess.) A. Robyns (Malvaceae), em Caboclo, Afrânio, Pernambuco. Comportamento: PE - Polinizador Efetivo, PO - Polinizador Ocasional e PI - Pilhador. Table 2 - Floral visitors of Pseudobombax marginatum (A. St.-Hil., Juss. \& Cambess.) A. Robyns (Malvaceae), in Caboclo, Afrânio, Pernambuco. Behavior: PE - Effective Pollinator, PO - Casual Pollinator and PI- Lacernists.

\begin{tabular}{llc}
\hline Família & Espécie & Comportamento \\
\hline Sphingidae & Agrius cingulata (Fabricius, 1775) & PE \\
& Protambulyx strigilis (Linnaeus, 1771) & PE \\
Noctuidae & Indeterminada & PI \\
Apidae & Apis mellifera (Linnaeus, 1758) & PI \\
& Trigona spinipes (Fabricius, 1793) & PI \\
& Xylocopa (Neoxylocopa) grisescens (Lepeletier, 1841) & PO/PI \\
Formicidae & Camponotus sp. & PI \\
Vespidae & Polybia ruficeps xanthops (Richards, 1978) & PI \\
Ave & Eupetomena macroura (Gmelin, 1788) & PI \\
\hline
\end{tabular}

Visitantes florais

As flores dos indivíduos de $P$. marginatum receberam visitas de himenópteros, lepidópteros e aves (Tab. 2).

Durante as observações noturnas foram registradas visitas dos esfingídeos Agrius cingulata (Fabricius 1775) e Protambulyx strigilis (Linnaeus 1771) e pequenas mariposas da Família Noctuidae.

No período diurno, foram registradas visitas das abelhas das espécies Trigona spinipes (Fabricius 1793), Apis mellifera (Linnaeus 1758) e Xylocopa (Neoxylocopa) grisescens (Lepeletier 1841), vespas da espécie Polybia ruficeps xanthops (Richards 1978) e o beija-flor Eupetomena macroura (Gmelin 1788).

Formigas do gênero Camponotus patrulharam as flores e botões florais durante todo período da antese realizando apenas pilhagem de néctar (Fig. 1b).

A visita do beija-flor Eupetomena macroura foi registrada apenas uma vez durante o estudo, próximo ao anoitecer. Na ocasião contatou apenas uma flor abordando-a frontalmente, não sendo registrada a deposição de pólen no corpo.

As abelhas $A$. mellifera e $T$. spinipes realizaram suas visitas entre $7 \mathrm{~h}$ e $8 \mathrm{~h}$ da manhã, abordando as flores frontalmente, agarrando-se nas anteras e coletando pólen e néctar (Fig. 1c,f). Apesar da coleta de pólen ser feita frontalmente, em nenhum momento foi registrado o contato das abelhas ao estigma. Durante as visitas, $T$. spinipes utilizou suas mandíbulas para realizar perfurações nas pétalas e cortar alguns estames para facilitar o acesso à câmara nectarífera (Fig. 1f).
Xylocopa (Neoxylocopa) grisescens iniciou as visitas por volta das $4 \mathrm{~h} 20 \mathrm{~min}$, tendo a última visita registrada às $8 \mathrm{~h}$. Contactou cerca de quatro flores por visita. Na coleta de pólen, agarraram-se às anteras e com as pernas traseiras realizaram movimentos de raspagem. Na coleta de néctar introduziram a cabeça entre os filetes para acessar o nectário, e nas duas ocasiões em que isto ocorreu, tocaram esporadicamente o estigma promovendo a polinização (Fig. 1d).

Agrius cingulata e $P$. strigilis, dentre os visitantes florais, foram os mais freqüentes às flores de P. marginatum (Fig. 2). Ambos iniciaram as visitas por volta das $17 \mathrm{~h} 50 \mathrm{~min}$, sendo a última visita registrada às $23 \mathrm{~h}$. Contataram cerca de seis flores de um mesmo indivíduo por visita. Ao

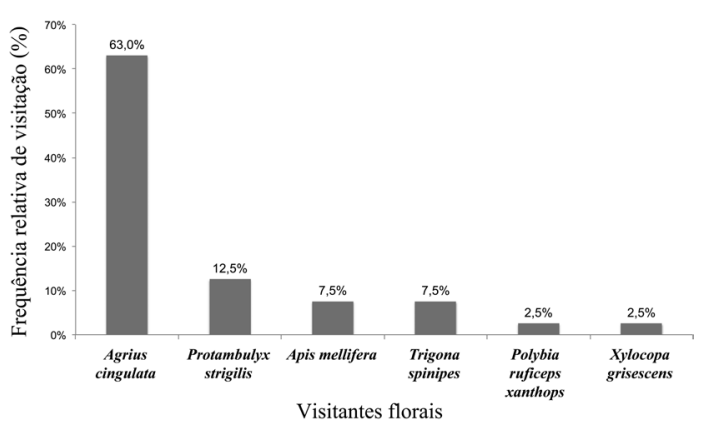

Figura 2 - Frequência de visitantes florais de Pseudobombax marginatum (A. St.-Hil., Juss. \& Cambess.) A. Robyns (Malvaceae), em Caboclo, Afrânio, Pernambuco.

Figure 2 - Frequency of floral visitors Pseudobombax marginatum (A. St.-Hil., Juss. \& Cambess.) A. Robyns (Malvaceae), in Caboclo, Afrânio, Pernambuco. 
Tabela 3 - Resultados dos experimentos do Sistema reprodutivo em Pseudobombax marginatum (A. St.-Hil., Juss. \& Cambess.) A. Robyns (Malvaceae), em uma área de Caatinga em Caboclo, Afrânio, Pernambuco.

Table 3 - Results of the breeding system experiments in Pseudobombax marginatum (A. St.-Hil., Juss. \& Cambess.) A. Robyns (Malvaceae), in an area of Caatinga in Caboclo, Afrânio, Pernambuco.

\begin{tabular}{lccc}
\hline Experimento & Flores (n) & Frutos (n) / \% & Sementes / Frutos (n) \\
\hline Controle & 30 & 0 & 0 \\
Pol. Cruzada & 16 & $6 / 37,5$ & 257,5 \\
Agamospermia & 30 & 0 & 0 \\
Autopol. espontânea & 16 & 0 & 0 \\
Autopol. manual & 15 & 0 & 0 \\
\hline
\end{tabular}

abordar as flores em visitas legítimas, introduziamse entre os estames para acessar o nectário, dessa forma contataram ligeiramente os estames e estigma (Fig. 1a,e). O pólen liberado foi depositado na parte ventral do corpo destes visitantes.

\section{Sistema reprodutivo}

Apenas no tratamento de polinização cruzada ocorreu formação de frutos $(37,5 \% ; \mathrm{n}=16)$, com $257,5 \pm 101,12$ sementes/fruto (Tab. 3). Apesar da produção de frutos observada sob condições naturais, 17,04 $\pm 17,92$ frutos/indivíduo $(\mathrm{n}=23$ indivíduos), com 217,44 $\pm 51,47$ sementes/frutos $(\mathrm{n}=10)$, não foi observado formação de frutos no teste controle $(\mathrm{n}=30)$. Nos demais tratamentos, também não houve formação de frutos, sugerindo que a espécie é auto-incompatível (Tab. 3).

\section{Discussão}

A floração anual e estratégia fenológica do tipo disponibilidade regular (Gentry 1974) observadas em Pseudobombax marginatum, são típicas de plantas quiropterófilas, sendo também observado em $P$. grandiflorum (Cav.) A. Robyns e Pachira calophylla (K.Schum.) Fern. Alonso (Fischer et al. 1992), ambas Bombacoideae. Essa estratégia pode aumentar a reprodução de Pseudobombax marginatum, pois no período do estudo não foi observada nenhuma espécie de antese noturna em floração. Desta maneira, esta espécie caracterizava a única fonte de recursos no período para os animais visitantes, sendo a possível consequência dos processos de evitar a competição com outras plantas por polinizadores, ou até mesmo pela escassez de recursos necessários a floração de outras espécies.

O expressivo volume e a baixa concentração de néctar produzido por $P$. marginatum também foi observado em outras espécies da subfamília
Bombacoideae, como Ceiba petandra, Pachira calophylla, Pseudobombax grandiflorum, Pseudobombax ellipticoideum e Pseudobombax marginatum (Eguiarte et al. 1987; Fischer et al. 1992; Gribel et al. 1999), Alta produção de néctar e a baixa concentração do mesmo podem resultar em um maior número de visitas a flores de indivíduos distintos, uma vez que a maior obtenção de energia pelo seu consumo dependerá da ingestão de um maior volume, consequentemente levando a um maior sucesso na polinização da espécie (Fischer \& Leal 2006). Estas características na produção e concentração do néctar estão associadas a polinização por morcegos (Faegri \& Pij1 1979).

Além das características da produção e concentração do néctar, citadas anteriormente, os atributos florais de Pseudobombax marginatum, tais como, flores grandes, com antese noturna, cores alvas, ausência de guias de néctar e distanciamento entre as anteras e a fonte de néctar, são comuns a flores de espécies quiropterófilas e esfingófilas (Faegri \& Pijl 1979). Contudo, algumas características como expressiva quantidade de néctar e pólen, odor desagradável, flores grandes com antese explosiva e néctar de baixa concentração, trazem a possibilidade de morcegos serem os polinizadores naturais de Pseudobombax marginatum, apesar de neste estudo esses visitantes não terem sido observados (Baker et al. 1998; Faegri \& Pijl 1979). A imediata produção de néctar associado ao forte odor liberado pelas flores de Pseudobombax marginatum, permite a criação de rotas de forrageamento "trap lining", que estão também relacionadas à polinização por morcegos, contudo a produção de várias flores por dia pode diminuir a distância das rotas de forrageamento dos morcegos (Gribel et al. 1990), uma vez que flores de um mesmo indivíduo podem ser visitadas várias vezes. 
A ausência de visita de quirópteros às flores de P. marginatum na localidade de Caboclo, pode estar relacionada à fragmentação florestal observada no local do estudo, uma vez que morcegos, depois de mamíferos não voadores, são o grupo de polinizadores com maior fragilidade ao processo de modificação da paisagem natural (Renner 1998; Aizen \& Feinsinger 2003; Fahrig 2003; Cavallero et al. 2013). Quesada et al. (2003) demonstraram o efeito da fragmentação florestal sobre árvores isoladas apontando como principal causa da redução do número de visitas dos polinizadores de espécies de Bombacoideae. Estudos apontam que esse processo é a principal causa da diminuição da riqueza e abundância de espécies polinizadoras, e consequentemente redução da qualidade dos serviços de polinização, alterando a guilda de polinizadores e diretamente prejudicando o fluxo gênico em populações de plantas (Aizen \& Feinsinger 1994; Machado et al. 1998; Johnson et al. 2004; Siqueira Filho \& Machado 2006; Sabatino et al. 2010), o que pode estar ocorrendo com P. marginatum na área. Este argumento ganha forças com a observação dos resultados obtidos neste estudo da baixa formação de frutos por polinização natural, provavelmente relacionada a carência de polinizadores específicos, neste caso os morcegos.

Apesar das características florais favorecerem a visita de animais de hábitos noturnos, foram observados também animais diurnos visitando as flores de $P$. marginatum. Este fato já foi reportado para espécies de Bombacoideae e outras espécies arbóreas de antese noturna que apresentam em comum flores do tipo pincel, que apresentam morfologia permissiva, podendo levar a um maior espectro de visitantes (Kuhlmann \& Kuhn 1947; Gribel et al. 1999; Duarte 2006; Cruz-Neto et al. 2007; Amorim et al. 2013; Barros et al. 2013; Avila et al. 2015). Essa estratégia favoreceu algumas espécies em outros estudos, uma vez que a extensão da antese e oferta de recurso por longos períodos deve aumentar o espectro de animais visitantes, consequentemente elevar o sucesso reprodutivo (Oliveira et al. 1992; Gribel et al. 1999; Barros et al. 2013), não sendo possível afirmar o mesmo para $P$. marginatum.

O comportamento dos visitantes em visitar várias flores de um mesmo indivíduo, eleva a geitonogamia, que geneticamente assemelha-se a autogamia (Bawa 1974), e consequentemente contribui com a baixa formação de frutos por $P$. marginatum, por ser autoincompatível. A deposição de pólen incompatível no estigma através dos polinizadores faz com que a planta selecione geneticamente grãos compatíveis e descarte as flores que receberam grãos das próprias flores e de doadores parentais próximos (Barros et al. 2013). Neste sentido, a ausência de morcegos como polinizadores pode estar ocasionando a baixa formação de frutos em $P$. marginatum, uma vez que este grupo de polinizadores podem apresentar comportamento de forrageio do tipo trap-lining, evitando ou diminuindo as taxas de geitonogamia, diferentemente de esfingídeos, abelhas e vespas que apresentam rotas de forrageio mais curtas (Koptur 1984; Richards 1997; Cruz-Neto et al. 2007; Barros et al. 2013).

A autoincompatibilidade encontrada para $P$. marginatum já havia sido observada em outras espécies de Bombacoideae, como Ceiba speciosa (A.St.-Hil.) Ravenna (Gibbs \& Bianchi 1993) e Ceiba pentandra (Lobo et al. 2005). Esse mecanismo pode ter contribuído para a baixa frutificação natural, já que obrigatoriamente deveria ocorrer a xenogamia, aumentando ainda mais a dependência da eficiência dos serviços de polinização. Segundo Bawa \& Webb (1984) a baixa formação de frutos é um fenômeno aparentemente comum nas angiospermas, tendo como uma das hipóteses a ausência de polinizadores eficientes causando a limitação polínica, o que pode estar ocorrendo com $P$. marginatum, já que a formação de frutos no tratamento de polinização cruzada foi maior que o observado na polinização natural (Harder \& Aizen 2010; Freitas et al. 2010).

A ausência de morcegos, indicados pelas características morfológicas das flores como polinizadores efetivos, unida a ocorrência de um sistema reprodutivo dependente do polinizador e presença de mecanismos de incompatibilidade, podem contribuir ainda mais para a diminuição do sucesso reprodutivo de $P$. marginatum, por conduzirem a espécie a limitação polínica (Faegri \& Pijl 1979; Ashman et al. 2004; Harder \& Aizen 2010; Freitas et al. 2010). Isto aliado ao processo de fragmentação florestal ocorrente na área de estudo colocam a espécie em uma situação crítica quanto à manutenção e regeneração de suas populações naturais, uma vez que esta modificação na paisagem natural tende a reduzir populações de polinizadores adequados, neste caso os morcegos, que apresentam grande sensibilidade a este processo (Renner 1998; Aizen \& Feinsinger 2003; Fahrig 2003; Quesada et al. 2003; Cavallero et al. 2013). 


\section{Agradecimentos}

À Fundação $\mathrm{O}$ Boticário de Proteção à Natureza (FBPN/PICN n ${ }^{\circ}$ 0673-2005.2), a bolsa concedida e o auxílio financeiro; a F.S.E. Santo e C.L. Seido, o auxílio nos trabalhos de campo; à Dra. Ana Luiza Du Bocage-Neta, Empresa Pernambucana de Pesquisa Agropecuária (IPA), a identificação de Pseudobombax; e à Dra. Catarina da Silva Motta, Instituto Nacional de Pesquisas da Amazônia (INPA), a identificação dos Esfingídeos.

\section{Referências}

Aizen, M.A. \& Feinsinger, P. 1994. Forest fragmentation, pollination, and plant reproduction in a Chaco dry forest, Argentina. Ecology 75: 330-351.

Aizen, M.A. \& Feinsinger, P. 2003. Bees not to be? Responses of insect pollinator faunas and flower pollination to habitat fragmentation. In: How landscapes change. Springer Berlin Heidelberg, Berlin. Pp. 111-129.

Alverson, W.S.; Nyffeler, R.; Whitlock, B.; Bayer, C. \& Baum, D.A. 1999. Phylogenetic analysis of the core Malvales based on sequences of $n d h \mathrm{~F}$. American Journal of Botany 86: 1474-1486.

Amorim, F.W.; Galetto, L. \& Sazima, M. 2013. Beyond the pollination syndrome: nectar ecology and the role of diurnal and nocturnal pollinators in the reproductive success of Inga sessilis (Fabaceae). Plant biology 15: 317-327.

Ashman, T.L.; Knight, T.M.; Steets, J.A.; Amarasekare, P.; Burd, M.; Campbell, D.R.; Dudash, M.R.; Johnston, M.O.; Mazer, S.J.; Mitchell, R.J.; Morgan, M.T. \& Wilson, W.G. 2004. Pollen limitation of plant reproduction: ecological and evolutionary causes and consequences. Ecology 85: 2408-2421.

Avila, R.; Pinheiro, M. \& Sazima, M. 2015. The generalist Inga subnuda subsp. luschnathiana (Fabaceae): negative effect of floral visitors on reproductive success? Plant Biology 17: 728-733.

Baker, H.G.; Baker, I. \& Hodges, S.A. 1998. Sugar composition of nectars and fruits consumed by birds and bats in the tropics and subtropics. Biotropica 30: 559-586.

Barroso, G.M.; Guimarães, E.F.; Ichaco, C.L.F.; Costa, C.G. \& Peixoto, A.L. 1978. Sistemática de Angiospermas do Brasil. Universidade de São Paulo, São Paulo. 255p.

Baum, D.A.; Dewitt Smith, S.; Yen, A.; Alverson, W.S.; Nyffler, R.; Whitlock, B.A. \& Oldham, R.A. 2004. Phylogenetic relationships of Malvatheca (Bombacoideae and Malvoideae; Malvaceae sensu lato) as inferred from plastid DNA sequences. American Journal of Botany 91: 1863-1871.
Bawa, K.S. 1974. Breeding systems of tree species of a lowland tropical community. Evolution 28: 85-92.

Bawa, K.S. \& Webb, C.J. 1984. Flower, fruit and seed abortion in tropical forest trees: implications for the evolution of paternal and maternal reproductive patterns. American Journal of Botany 71: 736-751.

Barros, E.C.O.; Weber, L.C. \& Machado, I.C. 2013. Limitação de polinizadores e mecanismo de autoincompatibilidade de ação tardia como causas da baixa formação de frutos em duas espécies simpátricas de Inga (Fabaceae - Mimosoideae) na Amazônia Central. Rodriguésia 64: 037-047.

Carvalho-Sobrinho, J.G. \& Queiroz, L.P. 2011. Morphological cladistic analysis of Pseudobombax Dugand (Malvaceae, Bombacoideae) and allied genera. Revista Brasileira de Botânica 34: 197-209.

Cavallero, L.; Raffaele, E. \& Aizen, M.A. 2013. Birds as mediators of passive restoration during early post-fire recovery. Biological Conservation 158: 342-350

Cruz-Neto, O.; Lopes, A.V. \& Machado, I.C. 2007. Ecologia da polinização de Inga striata (Benth.) (Leguminosae-Mimosoideae) em um remanescente de Mata Atlântica no Nordeste do Brasil. Revista Brasileira de Biociências 5: 570-572.

Duarte, M.C. 2006. Diversidade de Bombacaceae Kunth no Estado de São Paulo. 2006. Dissertação de Mestrado. Instituto de Botânica da Secretaria de Estado do Meio Ambiente, São Paulo. 99p.

Eguiarte, L.; Martinez Del Rio, C. \& Arita, H. 1987. El Néctar y el Polen como Recursos: el papel ecológico de los visitantes a las flores de Pseudobombax ellipticum (H.B.K.) Dugand. Biotropica 19: 74-82.

EMBRAPA. 1999. Sistema brasileiro de classificação de solos. Embrapa Solos, Rio de Janeiro. 412p.

Esteves, G.L. 2005. Flora da Reserva Ducke, Amazonas, Brasil: Bombacaceae. Rodriguésia 56: 115-124.

Faegri, K. \& van der Pijl, L. 1979. The principles of pollination ecology. Pergamon Press, New York. $248 \mathrm{p}$.

Fahrig, L. 2003. Effects of habitat fragmentation on biodiversity. Annual Review of Ecology and Systematics 34: 487-515.

Fischer, E.A.; Jimenez, F.A. \& Sazima, M. 1992. Polinização por morcegos em duas espécies de Bombacaceae na Estação Ecológica de Juréia, São Paulo. Revista Brasileira de Botânica 15: 67-72.

Fischer, E. \& Leal, I.R. 2006. Effect of nectar secretion rate on pollination success of Passiflora coccinea (Passifloraceae) in the central Amazon. Brazilian Journal of Biology 66: 29-41.

Freitas, L.; Wolowski, M. \& Sigiliano, M.I. 2010. Ocorrência de limitação polínica em plantas de Mata Atlântica. Oecologia Australis 14: 251-265. 
Galen, C. \& Plowright, R.C. 1987. Testing the accuracy of using peroxidase activity to indicate stigma receptivity. Canadian Journal of Botany 65: 107111.

Gentry, A.H. 1974. Flowering phenology and diversity in tropical Bignoniaceae. Biotropica 6: 64-68.

Gibbs, P.E. \& Bianchi, M. 1993. Post-pollination events in species of Chorisia (Bombacaceae) and Tabebuia (Bignoniaceae) with late-acting self-incompatibility. Botanica Acta 106: 64-71.

Goldenberg, R. \& Shepherd, G.J. 1998. Studies on the reproductive biology of Melastomataceae in "cerrado" vegetation. Plant Systematics and Evolution 211: 13-29.

Gribel, R. 1988. Visits of Caluromys lanatus (Didelphidae) to flowers of Pseudobombax tomentosum (Bombacaceae): a probable case of pollination by marsupials in Central Brazil. Biotropica 20: 344-347.

Gribel, R.; Sazima, I. \& Sazima, M. 1990. Flores pedem morcegos. Ciência Hoje 61: 22-28.

Gribel, R.; Gibbs, P.E. \& Queiróz, A.L. 1999. Flowering phenology and pollination biology of Ceiba pentandra (Bombacaceae) in Central Amazon. Journal of Tropical Ecology 15: 247-263.

Gribel, R. \& Gibbs, P.E. 2002. High outbreeding as a consequence of selfed ovule mortality and single vector bat pollination in the amazonian tree Pseudobombax munguba (Bombacaceae). International Journal Plant Sciences 163: 10351043.

Harder, L.D \& Aizen, M.A. 2010. Floral adaptation and diversification under pollen limitation. Philosophical Transactions the Royal Society B 365: 529-543.

Johnson, S.D.; Collin, C.; Wissman, J.; Halverson, E. \& Ågren, J. 2004. Factors influencing variation in seed production among populations of the endangered daisy Gerbera aurantiaca. Biotropica 36: 148-155.

Koptur, S. 1984. Outcrossing and pollinator limitation of fruit set: Breeding systems of neotropical Inga trees (Fabaceae: Mimosoideae). Evolution 38: 1130-1143.

Kuhlmann, M. \& Kuhn, E. 1947. A flora do distrito de Ibiti, município de Amparo. Secretaria da Agricultura do Estado de São Paulo, Instituto de Botânica (publicação da série B), São Paulo. 221p.

Lennartsson, T. 2000. Extinction thresholds and disrupted plant-pollinator interactions in fragmented plant populations. Ecology 83: 3060-3072.

Lobo, J.A.; Quesada, M. \& Stoner, K.E. 2005. Effects of pollination by bats on the mating system of Ceiba pentandra (Bombacaceae) populations in the two tropical life zones in the Costa Rica. American Journal of Botany 92: 370-376.

Machado, I.C. \& Lopes, A.V. 2003. Recursos florais e sistemas de polinização e sexuais em Caatinga. In:
Leal, I.R.; Tabarelli, M. \& Silva, J.M.C. Ecologia e Conservação da Caatinga. Editora Universitária da UFPE, Recife. Pp. 515-563.

Machado, I.C.; Sazima, I. \& Sazima, M. 1998. Bat pollination of the terrestrial herb Irlbachia alata (Gentianaceae) in northeastern Brazil. Plant Systematics and Evolution 209: 231-237.

MMA - Ministério do Meio Ambiente. 2002. Biodiversidade brasileira: avaliação e identificação de áreas prioritárias para conservação, utilização sustentável e repartição de benefícios da biodiversidade brasileira. MMA, Secretaria de Biodiversidade e Florestas, Brasília. 31p.

Newstrom, L.E.; Frankie, G.W. \& Baker, H.G. 1994. A new classification for plant phenology based on flowering patterns in lowland tropical rain forest trees at La Selva, Costa Rica. Biotropica 26: 141159.

Oliveira, P.E.; Gibbs, P.E.; Barbarosa, A.A. \& Talavera, S. 1992. Contrasting breeding systems in two Eriotheca (Bombacaceae) species of the Brazilian cerrados. Plant Systematics and Evolution 179: 207-219.

Quesada, M.; Stoner, K.E.; Rosas-Guerrero, V.; PalaciosGuevara, C. \& Lobo, J.A. 2003. Effects of habitat disruption on the activity of nectarivorous bats (Chiroptera: Phyllostomidae) in a dry tropical forest: implications for the reproductive success of the neotropical tree Ceiba grandiflora. Oecologia 135: 400-406.

Renner, S.S. 1998. Effects of habitat fragmentation on plant pollinator interactions in the tropics. In: Newbery, D.M.; Prins, H.H.T. \& Brown, N.D. Dynamics of tropical communities. Blackwell Science, New Jersey. Pp. 339-360.

Robyns, A. 1963. Essai de Monographie du genre Bombax L. s.l. (Bombacaceae). Bulletin du Jardin Botanique de l'État 33: 1-315.

Sabatino, M.; Maceira, N. \& Aizen, M.A. 2010. Direct effects of habitat area on interaction diversity in pollination webs. Ecological Applications 20: 1491-1497.

Silva, S.S.P. \& Peracchi, A.L. 1995. Observação da visita de morcegos (Chiroptera) às flores de Pseudobombax grandiflorum (CAV.) A. Robyns. Revista Brasileira de Zoologia 12: 859-865.

Siqueira-Filho, J.A.; Santos, A.P.B.; Nascimento, M.F.S \& Santo, F.S.E. 2009. Guia de campo de árvores da caatinga. Editora e Gráfica Franciscana LTDA, Petrolina. 64p.

Siqueira-Filho, J.A. \& Machado, I.C. 2006. Floração e polinização das bromélias na Mata Atlântica nordestina. In: Siqueira-Filho, J.A. \& Leme, E.M.C. Fragmentos da Mata Atlântica do Nordeste: biodiversidade, conservação e suas bromélias. Andrea Jakobson Estúdio Editorial, Rio de Janeiro. Pp. 159-189. 
Souza, V.C. \& Lorenzi, H. 2012. Botânica Sistemática: guia ilustrado para identificação das famílias de Fanerógamas nativas e exóticas no Brasil, baseado em APG III. $3^{\text {a }}$ ed. Instituto Plantarum, Nova Odessa. 768p.

Teixeira, R.C. 2010. Partilha de polinizadores por espécies quiropterófilas em um fragmento de cerrado, São Paulo. Tese de Doutorado. Universidade Federal de São Carlos, São Paulo. 116p.

Velloso, A.L.; Sampaio, E.V.S.B.; Guilietti, A.M.; Barbosa, M.R.V.; Castro, A.A.J.F.; Queiroz, L.P.; Fernandes, A.; Oren, D.C.; Ceastro, L.A.; Carvalho, A.J.E.; Pareyn, F.G.C.; Silva, F.B.R.; Miranda, E.E.;
Keel, S. \& Gondim, R.S. 2002. Ecorregiões da Caatinga. In: Velloso, A.L.; Sampaio, E.V.S.B. \& Pareyn, F.G.C. Ecorregiões: propostas para o bioma Caatinga. APNE/TNC do Brasil, Recife. Pp. 7-34.

Yamamoto, L.F.; Kinoshita, L.S. \& Martins, F.R. 2007. Síndromes de polinização e de dispersão em fragmentos da Floresta Estacional Semidecídua Montana, SP, Brasil. Acta Botanica Brasilica 21: 553-573.

Zortéa, M. 2003. Reproductive patterns and feeding habits of three nectarivorous bats (phyllostomidae: Glossophaginae) from the brazilian cerrado. Brazilian Journal Biology 63: 159-168. 